\title{
The Direct Growth of Carbon Nanotubes as Vertical Interconnects in 3D Integrated Circuits
}

\author{
Sten Vollebregt*a ${ }^{*}$, Ryoichi Ishihara ${ }^{a}$ \\ ${ }^{a}$ Delft University of Technology, Else Kooi Laboratory, Faculty of Electrical Engineering, \\ Mathematics and Computers Science, Laboratory of Electronic Components, Technology \\ and Materials, Feldmannweg 17, 2628 CT, Delft, The Netherlands, E-mail: \\ s.vollebregt@tudelft.nl
}

\begin{abstract}
Carbon nanotubes (CNT) have been proposed for many applications in integrated circuits (IC): ranging from transistors and interconnects to sensors and actuators. For these applications it is crucial to integrate CNT directly alongside electronics, something which has not been achieved before. In this work we demonstrate the direct growth of CNT alongside CMOS devices, by integrating CNT as vertical interconnect (vias) in a monolithic 3D IC process using techniques and materials compatible with semiconductor technology. The electrical performance of both the CNT vias and the electrical devices is investigated and compared with the literature. From this we can conclude that the CNT growth has no significant impact on the electrical devices, although the resistance of the CNT should be further reduced to compete with metal interconnects. This demonstrates the viability of integrating CNT with IC, which is an important step forward in the application of CNT in electronics.
\end{abstract}

* Corresponding author. Tel. +3115 2786788; Fax +3115 2622163; E-mail address: 


\section{Introduction}

Due to their fascinating physical properties carbon nanotubes (CNT) have attracted extensive attention for applications in integrated circuits (ICs). Potential application of vertically aligned CNTs are as transistors,[1] sensors,

field-emission devices, [2] thermal management material,[3], super-capacitors,[4] and vertical interconnects.[5, 1]. For the latter this is because of the excellent thermal conductivity of CNT up to $3500 \mathrm{~W} / \mathrm{mK}$ at room temperature,[6] their bottom-up nature of fabrication resulting in high aspect ratios, and their high current carrying capability up to 3 orders in magnitude higher than that of Cu.[7]

All of these applications require CNT to be positioned accurately at predefined locations, while not damaging the already fabricated (electronic) structures. CNT can be placed on integrated circuits using pick and place techniques after fabrication, in which case the CNT are grown separately, harvested and purified.[8, 9] Another possibility is the local heating of a chip using on-chip heaters for selective CNT growth.[10] However, such techniques are regarded as cumbersome and inaccurate, resulting in bad to none industrial scalability.[11]

Ideally one would directly deposit, that is grow, the CNT at the desired location. However, this gives rise to strong limitations in the maximum processing temperatures and the choice of materials in order to allow integration with existing semiconductor fabrication techniques. Chemical vapour deposition (CVD) on top of transition metal catalyst nanoparticles is regarded

s.vollebregt@tudelft.nl (S. Vollebregt). 
as the most promising technique for the direct growth of CNT on electronics, as it allows the lowest processing temperatures. Recently, our group has demonstrated growth of vertically aligned CNT at temperatures as low as $350^{\circ} \mathrm{C},[12]$ which is regarded as a CMOS compatible temperature. From a material perspective Fe should be avoided as catalyst for the CVD process, as it is regarded as a contaminant in IC fabrication.[13] Finally, for the application as electrical conductor for interconnects and transistors, but also for sensors, field emission devices and integrated super-capacitors, CNT should be grown on an electrically conducting substrate or support layer. This, in order to minimize the electrical contact resistance to the CNT.

While many examples are available of growing vertical CNT at moderate to low temperatures using CVD for the fabrication of CNT test vias, [5, 14, $15,16,17,18,19,12]$ or thermal interface materials, [20, 21] none of these examples demonstrates the possibility of growing CNT directly on existing electronic circuits. In this work we integrated CNT, grown using Co on TiN support layers, as vertical interconnects into a monolithic 3D IC process in order to demonstrate the possibility of the direct integration of CNT in IC. Monolithic 3D IC are seen as the ultimate 3D integration approach allowing the extension of Moore's law and combining different technologies within the same chip with maximum bandwidth.[22] CNT were chosen as via material in this process, as monolithic 3D IC will require via aspect ratios not achievable with the current metallization schemes available in IC fabrication.

With this combined CNT and 3D IC technology, individual transistors, CMOS inverters and 6 transistor static random access memory (6T-SRAM) cells were made and electrically characterised. We have been able to con- 
struct CNT vias with aspect ratios as high as 2.6, by fabricating vias $1 \mu \mathrm{m}$ in diameter and with a length of $2.6 \mu \mathrm{m}$. This is already higher than that of metallization schemes with $\mathrm{Cu}$, which is currently limited to 1.9 according to the International Technology Roadmap for Semiconductors.[23] More importantly we demonstrate the possibility of fabricating CNT directly on already fabricated electronics, which is a leap forward in the utilization of CNT for a broad range of electronic applications.

\section{Experimental}

The active devices were fabricated by the $\mu$-Czochralski method, which is described in more details elsewhere.[24, 25] The process, as illustrated in Supplementary Figure 2, starts with the definition of $1 \mu \mathrm{m}$ wide holes in a $750 \mathrm{~nm}$ thick layer of thermal $\mathrm{SiO}_{2}$. These holes are then filled by $830 \mathrm{~nm}$ of $\mathrm{SiO}_{2}$ deposited using plasma-enhanced chemical vapour deposition (PECVD), leaving holes of $\sim 100 \mathrm{~nm}$ called grain filters. The wafers are then covered by $250 \mathrm{~nm}$ of a-Si deposited at $545{ }^{\circ} \mathrm{C}$ with low-pressure chemical vapour deposition (LPCVD). With an excimer laser the a-Si is locally melted, which results in the formation of single-crystal Si grains with an area of 6 by $6 \mu \mathrm{m}$ from the grain filters.

After crystallization, the Si transistor islands are defined by dry etching, followed by the deposition of a $50 \mathrm{~nm}$ PECVD gate oxide and LPCVD a-Si gate. After gate patterning, $\mathrm{Ar}^{+}$and $\mathrm{B}^{+}$implantations are performed and activated with excimer laser annealing. In the next step, the transistors are covered by $600 \mathrm{~nm}$ PECVD $\mathrm{SiO}_{2}$, and then the contact openings to the gate and Si islands are defined. Inside these openings a stack of 10/50/100 nm of 
$\mathrm{Ti} / \mathrm{TiN} / \mathrm{Ti}$ is deposited. The first layer of $\mathrm{Ti}$ acts as an adhesion layer, the TiN as a support layer for the catalyst used to grow the CNT, and the last $\mathrm{Ti}$ as a sacrificial layer to protect the TiN layer from plasma damage.[26]

To finalize the first transistor layer, a $1.2 \mu \mathrm{m}$ thick $\mathrm{SiO}_{2}$ layer is deposited, in which $750 \mathrm{~nm}$ deep holes are etched for the second layer grain filter. Again, these holes are filled by $830 \mathrm{~nm}$ of PECVD $\mathrm{SiO}_{2}$, after which the second layer transistors are made by applying the same procedure as used for the first layer. After the implantation and dopant activation steps, the second layer transistors are covered by $600 \mathrm{~nm}$ of PECVD $\mathrm{SiO}_{2}$, and contact openings to the second layer gate and Si islands are defined. Into these openings a stack of $10 / 50 \mathrm{~nm}$ of $\mathrm{Ti} / \mathrm{TiN}$ is deposited, in order to prevent the formation of native $\mathrm{SiO}_{2}$.

The via openings to the first layer (through $2.5 \mu \mathrm{m}$ of oxide) are then dry etched, followed by wet removal of the $100 \mathrm{~nm}$ Ti sacrificial layer in a $0.55 \% \mathrm{HF}$ solution for 1.5 minutes. Using the same resist as used for the via hole definition, a $5 \mathrm{~nm}$ thick layer of Co is evaporated into the openings and patterned by lift-off with tetrahydrofuran as the solvent. Next, CNT are grown inside the via openings using a commercially available AIXTRON Blackmagic Pro LPCVD reactor at $500{ }^{\circ} \mathrm{C}, 8 \mathrm{kPa}$, and with $700 / 50 \mathrm{sccm}$ $\mathrm{H}_{2} / \mathrm{C}_{2} \mathrm{H}_{2}$ for 1:50 minutes. Finally, a $100 \mathrm{~nm}$ of $\mathrm{Ti}$ and a $2 \mu \mathrm{m}$ of $\mathrm{Al}(1 \%$ $\mathrm{Si}$ ) layer stack is sputtered to connect the vias and second layer transistors. This metal layer is subsequently patterned by dry etching.

Measurements were performed using an Agilent E5270B parameter analyser on a semi-automatic probe station with temperature controlled chuck. SEM images were obtained with a Philips XL50 electron microscope. 


\section{Results and discussion}

We now discuss the results of integrating CNT with IC technology, and the impact these technologies have on each other. In Figure 1 scanning electron microscopy (SEM) images taken of CNT vias before a) and after b), c) final top metallization, as described in the methods section, can be found. The SEM image which was taken before metallization clearly show the top transistor, and the $\mathrm{CNT}$ vias filling the, in this case, $2 \mu \mathrm{m}$ wide vias. In Supplementary Figure 1 a top view of an array of $1 \mu \mathrm{m}$ wide CNT vias can be found, demonstrating the uniformity of the process. Figure $1 \mathrm{~b}$ ) displays a detailed cross-section of a $2 \mu \mathrm{m}$ wide CNT via, with an approximate CNT density of $5 \cdot 10^{10}$ tubes $/ \mathrm{cm}^{2}$, estimated by counting. The bright region around the bottom of the via opening is the $\mathrm{Ti} / \mathrm{TiN}$ stack used as support layer for successful catalyst activation.[26] The $2.6 \mu \mathrm{m}$ height of the CNT bundles is the same as the depth of the via opening and the removed Ti sacrificial layer which was used for protecting the TiN against plasma damage.

The cross-section in Figure 1 c) shows two transistors on top of each other (marked by red boxes). As no chemical mechanical polishing was performed, the top-layer transistor is slightly curved by the oxide layers underneath. This cross-section was made from elongated two-layer transistor designed to facilitate easy cleaving of the sample for cross-section preparation. However, when growing CNT in a long line they become wavelike over the length of the cavity, instead of a solid cube as shown in Figure $1 \mathrm{~b}$ ). This explains the deformation of the CNT bundle observed in Figure $1 \mathrm{c}$ ).

Co was used in this work, as Fe was found to result in random growth on TiN which had been exposed to excimer laser crystallization and LPCVD 
amorphous Si deposition. We assume that the process steps, required for our 3D IC fabrication, alter the properties of the TiN layer, and therefore change the activation of the catalyst into nano-islands and the subsequent growth of the CNT. One advantage of Co is that it is considered to be cleanroom compatible, and is often used for producing source/drain silicides. Moreover, we recently showed that Co can be used to grow CNT at temperatures as low as $350^{\circ} \mathrm{C},[12]$ which would make the process compatible with advanced low- $\kappa$ dielectrics and $\mathrm{Cu}$ metallization.

Beside the active devices which will be discussed later, the wafer contained four-point probe measurement structures with different CNT via diameters in order to characterize the CNT via resistance to the first transistor layer gate and source/drains area. Current-Voltage (I-V) measurements were performed at different temperatures on these structures in order to investigate the individual via resistance and their thermal coefficient of resistance (TCR).

The CNT test via resistivities are compared in Figure 2 to values obtained before from test vias without active devices integrated alongside, and values from the literature.[5, 14, 15, 16, 17, 18, 19, 12] The average resistivities are 41 and $48 \mathrm{~m} \Omega$-cm to the gate and the source/drain area, respectively. The vias have a resistivity that is two times larger as compared to the values obtained before by using Fe as the catalyst at a growth temperature of $500{ }^{\circ} \mathrm{C}$. This is likely caused by the change of catalyst, as Co gives a roughly 2 times lower density and slightly lower crystallinity as compared to Fe-grown tubes.[12] Clearly, integrating the CNT vias with the 3D IC process has no dramatic impact on the CNT properties as the resistivities are very close to values 
obtained before. Still, these values are significantly higher than that of bulk $\mathrm{Cu}(1.7 \mu \Omega-\mathrm{cm})$.

The resistances of CNT test vias to the gate and source/drain areas of the transistor measured at different wafer temperatures are plotted in Figure 3. It is shown that the vias have a negative TCR, which is consistent with results obtained before on Fe-grown CNT test vias with different dimensions.[27] The average TCR to the gate and source/drain levels are -420 and -460 ppm/K, respectively. No significant change of TCR with CNT via width was observed for the 1,2 , and $3 \mu \mathrm{m}$ wide vias tested here. The negative TCR originates from additional conduction bands opening up for elevated temperatures, which dominates over the increase in scattering.[28] This negative TCR is advantageous for reliable operation of CNT vias in integrated circuits, as it reduces the resistive losses for increasing temperature.

Figure 4 displays the $\mathrm{I}_{\mathrm{D}}-\mathrm{V}_{\mathrm{G}}$ characteristics of the bottom layer transistors with CNT vias of $1 \mu \mathrm{m} \mathrm{a}$ ), c) and $2 \mu \mathrm{m} \mathrm{b}$ ), d) width. The gate length of these transistors is $1.5 \mu \mathrm{m}$, and the widths are 2 and $4 \mu \mathrm{m}$ for NMOS and PMOS transistors, respectively. Excellent transistor characteristics with high field effect mobility were obtained for both the NMOS (up to $368 \mathrm{~cm}^{2} / \mathrm{Vs}$ ) and PMOS (up to $145 \mathrm{~cm}^{2} / \mathrm{Vs}$ ) transistors with $2 \mu \mathrm{m}$ wide vias. As can be seen in Supplementary Table 1 the first layer transistor characteristics are similar of those obtained before in our group, but with aluminium (Al) vias.[25] This demonstrates that the relative short $500{ }^{\circ} \mathrm{C}$ CNT growth step has no negative impact on the transistor performance.

For the $1 \mu \mathrm{m}$ wide vias the mobility of the transistors is, however, significantly lower. We found that for the minimum sized vias $\left(1 \mu \mathrm{m}^{2}\right.$, aspect ratio 
2.6) the relative high via resistance decreases the transistor performance in terms of on-current and therefore mobility (Figure 5a). This also resulted in a slight decrease of the on/off ratio for the NMOS transistors. Compared to the ideal transistor which has a substreshold slope (SS) close to $60 \mathrm{mV} / \mathrm{dec}$ the values here are relative high. The average SS are $270 \pm 70$ and $260 \pm 50$ for the PMOS and NMOS, respectively. Moreover, the $V_{\text {th }}$ has a negative shift from the desired value of $\pm 1.5 \mathrm{~V}:-2.1 \pm 0.5 \mathrm{~V}$ and $0.6 \pm 0.3 \mathrm{~V}$ for, respectively, the PMOS and NMOS. Both effects are caused by the PECVD deposition of the gate oxide, which is known to have a relative large positive surface charge and results in a relative large variation of these performance indicators over the wafer [29]. Switching to a higher quality low temperature oxide, like inductive-coupled plasma PECVD oxide, can prevent this as is shown in the literature [30]. We found no evidence for any influence of the CNT diameter on the (average) $\mathrm{V}_{\text {th }}$ (Figure 5b) and SS, indicating that the transistors themselves function as expected.

With the design shown in Figure 6, inverters in which both active areas are directly on top of each other were made. The PMOS transistor was twice the width of that of an NMOS transistor, respectively 4 and $2 \mu \mathrm{m}$, to account for the difference in mobility for electrons and holes. The gate length is again $1.5 \mu \mathrm{m}$. Both configurations with the PMOS as top and as bottom transistor were fabricated on the wafer, each with vias widths of 1 , 2 and $3 \mu \mathrm{m}$. Using a $\mathrm{V}_{\text {DD }}$ of $5 \mathrm{~V}$ the $\mathrm{V}_{\text {in }}-\mathrm{V}_{\text {out }}$ characteristics were measured, and mid-point voltage $\left(\mathrm{V}_{\mathrm{M}}\right)$, the voltage swing, and Voltage Noise Margins 
(VNM) were extracted. The VNM are defined as:

$$
\begin{array}{r}
\mathrm{VNM}_{\mathrm{H}}=\mathrm{V}_{\mathrm{dd}}-\mathrm{V}_{\mathrm{IH}} \\
\mathrm{VNM}_{\mathrm{L}}=\mathrm{V}_{\mathrm{IL}}
\end{array}
$$

where $V_{I L}$ and $V_{I H}$ are defined as the input voltages for the points where the slope of the $V_{\text {in }}-V_{\text {out }}$ characteristics is -1 for an input equal to a logic ' 0 ' and '1', respectively (see Figure 7). For good stability large VNM are desired. Figure 7 displays the input-output characteristics of a PMOS bottom 3D inverter with minimal size CNT vias $\left(1 \mu \mathrm{m}^{2}\right.$, aspect ratio 2.6$)$ and full voltage swing.

The extracted inverter parameters can be found in table 1 for both the PMOS and NMOS-bottom inverters. For each size and type, 156 inverters were measured. Just as was the case for individual transistors, the smallest size vias have an impact on the performance of the inverters. From the perspective of $V_{M}$ being close to half of $V_{D D}$ and obtaining large $V N M$, a PMOS as the bottom transistor offers the best performance. This can be explained by looking at the threshold voltages of the transistors in Figure $5 \mathrm{~b})$, which are much larger in magnitude for the PMOS than the NMOS. This is especially the case for the top PMOS configuration, which makes the NMOS transistor switch much faster when the input potential is increased, subsequently lowering $\mathrm{V}_{\mathrm{M}}$ and $\mathrm{VNM}_{\mathrm{L}}$.

Beside the 3D inverters the wafer also contained 3D 6T-SRAM cells in which the NMOS access transistors and the pull-down network are in one device layer and the PMOS pull-up network in another, resulting in an area reduction compared to a 2D planar circuit up to $40 \%$. Only a few working SRAM cells were found on the wafer. This can be attributed to the transistor 
sizes used by the design, which were tailored for a traditional 3D IC process using $\mathrm{Al}$ as vias. The $\mathrm{Al}$ vias have a much lower resistivity compared to the CNT vias. Because of this, the balance between the pull-up, pull-down and access transistors is brought out of its design, resulting in non-working SRAM cells.

At a high $\mathrm{V}_{\mathrm{DD}}$ of $10 \mathrm{~V}$ the largest-sized SRAM cells were found operational. These devices have $24 \mu \mathrm{m}$ wide NMOS pull-down transistors in the bottom layer, $16 \mu \mathrm{m}$ wide PMOS pull-up transistors in the top layer, and either 2.6 or $4 \mu \mathrm{m}$ wide NMOS access transistors, again in the bottom layer. Figure 8 displays the butterfly diagram of the working 3D IC 6T-SRAM. The device has only very low readout-noise margins, judging from the small opening in the butterfly readout curves. Yet, it demonstrates that it is possible to fabricate more complex structures with the combined 3D IC and CNT process.

As mentioned before the CNT growth was not found to impact the functioning of the transistors, as similar mobilities, substreshold slopes and threshold voltages were obtained as compared with our earlier work on a 3D IC process with $\mathrm{Al}$ metallization for the vias [25]. However, the relative high CNT resistivity was found to limit the performance of both individual transistors as CMOS inverters in case of the smallest $1 \mu \mathrm{m}$ wide vias, as it becomes apparent from Figs. 4-5 and table 1. The crystallinity and density of the CNT bundles has to be increased drastically in order to be able to outperform Cu.[31, 32] Techniques have been proposed to increase the density of the bundles, for instance by using two-step catalyst deposition,[33] or using an Co-Mo alloy as catalyst.[34] The lowest resistivity obtained so far 
has been by Yokoyama et al.,[15] see also Figure 2, which was obtained by using a special catalyst deposition method using an impactor and a remote plasma CVD system.

\section{Conclusion}

We demonstrated the integration of CNT technology into a monolithic 3D IC technology as vertical interconnects with via aspect ratios as high as 2.6. Individual vias, transistors, 3D inverters and 6T-SRAM cells were made. The CNT vias showed a negative TCR and equal resistivity as compared to the values from the literature. This indicates that the $3 \mathrm{D}$ IC process has no significant impact on the CNT vias. Good functioning transistors were obtained; however, it was found that for the smallest size vias with an aspect ratio 2.6, the resistance decreases the performance. The $3 \mathrm{D}$ inverters, each with 3 CNT vias, demonstrated excellent performances, even though the smallest size vias again limited the characteristics. Although the high via resistance limits the performance, the characteristics of the transistors is not influenced by the CNT growth. This work demonstrates the possibility to integrate CNT with integrated circuits, without damaging these devices. This is an important step forward in the application of CNT for a broad range of applications in micro-electronics. The remaining challenge will be decreasing the resistivity of the CNT vias, in order to fully benefit from the advantages CNT can offer. 


\section{Acknowledgement}

The authors would like to thank the Else Kooi Lab staff (formerly known as Dimes TC) for processing support, and A. Sammak for checking the manuscript. A part of the work has been performed in the project JEMSiP_3D, which is funded by the Public Authorities in France, Germany, Hungary, The Netherlands, Norway and Sweden, as well as by the ENIAC Joint Undertaking.

[1] Robertson J. Growth of nanotubes for electronics. Materials Today 2007;10(1-2):36-43.

[2] Fan S, Chapline MG, Franklin NR, Tombler TW, Cassell AM, Dai H. Self-oriented regular arrays of carbon nanotubes and their field emission properties. Science 1999;283(5401):512-514.

[3] Huang H, Liu CH, Wu Y, Fan S. Aligned carbon nanotube composite films for thermal management. Advanced Materials 2005;17(13):16521656.

[4] Fiorentino G, Vollebregt S, Tichelaar FD, Ishihara R, Sarro PM. Impact of the atomic layer deposition precursors diffusion on solid-state carbon nanotube based supercapacitors performances. Nanotechnology 2015;26(6):064002-1-11.

[5] Kreupl F, Graham AP, Duesberg GS, Steinhögl W, Liebau M, Unger E, Hönlein W. Carbon nanotubes in interconnect applications. Microelectronic Engineering 2002;64(1-4):399-408. 
[6] Pop E, Mann D, Wang Q, Goodson K, Dai H. Thermal conductance of an individual single-wall carbon nanotube above room temperature. Nano Letters 2006;6(1):96-100.

[7] Wei BQ, Vajtai R, Ajayan PM. Reliability and current carrying capacity of carbon nanotubes. Applied Physics Letters 2001;79(8):1172-1174.

[8] Wei H, Patil N, Lin A, Wong HSP, Mitra S. Monolithic threedimensional integrated circuits using carbon nanotube FETs and interconnects. In: IEEE International Electron Devices Meeting. 2009, p. $1-4$.

[9] Patil N, Lin A, Myers ER, Ryu K, Badmaev A, Zhou C, Wong HSP, Mitra S. Wafer-scale growth and transfer of aligned single-walled carbon nanotubes. IEEE Transactions on Nanotechnology 2009;8(4):498-504.

[10] Santra S, Ali SZ, Guha PK, Zhong G, Robertson J, Covington JA, Milne WI, Gardner J, Udrea F. Post-cmos wafer level growth of carbon nanotubes for low-cost microsensors - a proof of concept. Nanotechnology 2010;21:485301.

[11] Duesberg GS, Graham AP, Kreupl F, Liebau M, Seidel R, Unger E, Hoenlein W. Ways towards the scaleable integration of carbon nanotubes into silicon based technology. Diamond and Related Materials 2004;13:354-361.

[12] Vollebregt S, Tichelaar FD, Schellevis H, Beenakker CIM, Ishihara R. Carbon nanotube vertical interconnects fabricated at temperatures as low as $350^{\circ} \mathrm{C}$. Carbon 2014;71:249-256. 
[13] Istratov A, Hieslmair H, ERWeber. Iron contamination in silicon technology. Applied Physics A 2000;70:489-534.

[14] Choi YM, Lee S, Yoon HS, Lee MS, Kim H, Han I, Son Y, Yeo IS, Chung UI, Moon JT. Integration and electrical properties of carbon nanotube array for interconnect applications. In: Sixth IEEE Conference on Nanotechnology. 2006, p. 262-265.

[15] Yokoyama D, Iwasaki T, Ishimaru K, Sato S, Hyakushima T, Nihei M, Awano Y, Kawarada H. Electrical properties of carbon nanotubes grown at a low temperature for use as interconnects. Japanese Journal of Applied Physics 2008;47(4):1985-1990.

[16] Dijon J, Okuno H, Fayolle M, Vo T, Pontcharra J, Acquaviva D, Bouvet D, Ionescu AM, Esconjauregui CS, Capraro B, Quesnel E, Robertson J. Ultra-high density carbon nanotubes on $\mathrm{Al}-\mathrm{Cu}$ for advanced vias. In: IEEE International Electron Devices Meeting. 2010, p. 33.4.1-4.

[17] Chiodarelli N, Masahito S, Kashiwagi Y, Li Y, Arstila K, Richard O, Cott DJ, Heyns M, Gendt SD, Groeseneken G, Vereecken PM. Measuring the electrical resistivity and contact resistance of vertical carbon nanotube bundles for application as interconnects. Nanotechnology 2011;22(8):085302.

[18] Vereecke B, van der Veen MH, Barbarin Y, Sugiura M, Kashiwagi Y, Cott DJ, Huyghebaert C, Tökei Z. Characterization of carbon nanotube based vertical interconnects. In: Extended Abstracts of the 2012 
International Conference on Solid State Devices and Materials. 2012, p. 648-649.

[19] van der Veen MH, Barbarin Y, Vereecke B, Sugiura M, Kashiwagi Y, Cott DJ, Huyghebaert C, Tökei Z. Electrical improvement of cnt contacts with $\mathrm{cu}$ damascene top metallization. In: IEEE International Interconnect Technology Conference. 2013, p. 193-195.

[20] Tong T, Zhao Y, Delzeit L, Kashani A, Meyyappan M, Majumdar A. Dense vertically aligned multiwalled carbon nanotube arrays as thermal interface materials. IEEE Transactions on Components and Packaging Technologies 2007;30(1):92-100.

[21] Zhang K, Chai Y, Yuen MMF, Xiao DGW, Chan PCH. Carbon nanotube thermal interface material for high-brightness light-emitting-diode cooling. Nanotechnology 2008;19(21):215706.

[22] Topol AW, DC La Tulipe J, Shi L, Frank DJ, Bernstein K, Steen SE, Kumar A, Singco GU, Young AM, Guarini KW, Ieong M. Threedimensional integrated circuits. IBM Journal of Research and Development 2006;50(4/5):491-506.

[23] ITRS. International technology roadmap for semiconductors. 2013. Http://public.itrs.net/.

[24] Mofrad MRT, Derakhshandeh J, Ishihara R, Baiano A, van der Cingel J, Beenakker K. Stacking of single-grain thin-film transistors. Japanese Journal of Applied Physics 2009;48:03B015. 
[25] Derakhshandeh J, Golshani N, Ishihara R, Mofrad MRT, Robertson M, Morrison T, Beenakker CIM. Monolithic 3-D integration of SRAM and image sensor using two layers of single-grain silicon. IEEE Transactions on Electron Devices 2011;58(11):3954-3961.

[26] Vollebregt S, Ishihara R, Derakhshandeh J, van der Cingel J, Schellevis $\mathrm{H}$, Beenakker CIM. Integrating low temperature aligned carbon nanotubes as vertical interconnects in si technology. In: 11th IEEE Conference on Nanotechnology. 2011, p. 985-990.

[27] Vollebregt S, Banerjee S, Beenakker CIM, Ishihara R. Size-dependent effects on the temperature coefficient of resistance of carbon nanotube vias. IEEE Transactions on Electron Devices 2013;60(12):4085-4089.

[28] Naeemi A, Meindl JD. Physical modeling of temperature coefficient of resistance for single- and multi-wall carbon nanotube interconnects. IEEE Electron Device Letters 2007;28(2):135-138.

[29] Ishihara R, Derakhshandeh J, Mofrad MT, Chen T, Golshani N, Beenakker C. Monolithic 3d-ics with single grain si thin film transistors. Solid-State Electronics 2012;71:80-87.

[30] Chen T, Ishihara R, Beenakker K. High quality sio2 deposited at 80 ${ }^{\circ} \mathrm{C}$ by inductively coupled plasma enhanced CVD for flexible display application. Electrochemical and Solid-State Letters 2010;13(8):89-91.

[31] Naeemi A, Meindl JD. Performance modeling for single- and multiwall carbon nanotubes as signal and power interconnects in gigascale systems. IEEE Transactions on Electron Devices 2008;55(10):2574-2582. 
[32] Li H, Srivastava N, Mao JF, Yin WY, Banerjee K. Carbon nanotube vias: Does ballistic electron-phonon transport imply improved performance and reliability. IEEE Transactions on Nanotechnology 2011;58(8):2689-2701.

[33] Esconjauregui S, Fouquet M, Bayer BC, Ducati C, Smajda R, Hofmann S, Robertson J. Growth of ultrahigh density vertically aligned carbon nanotube forests for interconnects. ACS Nano 2010;4(12):7431-7436.

[34] Sugime H, Esconjauregui S, Yang J, D'Arsié L, Oliver RA, Bhardwaj S, Cepek C, Robertson J. Low temperature growth of ultra-high mass density carbon nanotube forests on conductive supports. Applied Physics Letters 2013;103:073116. 

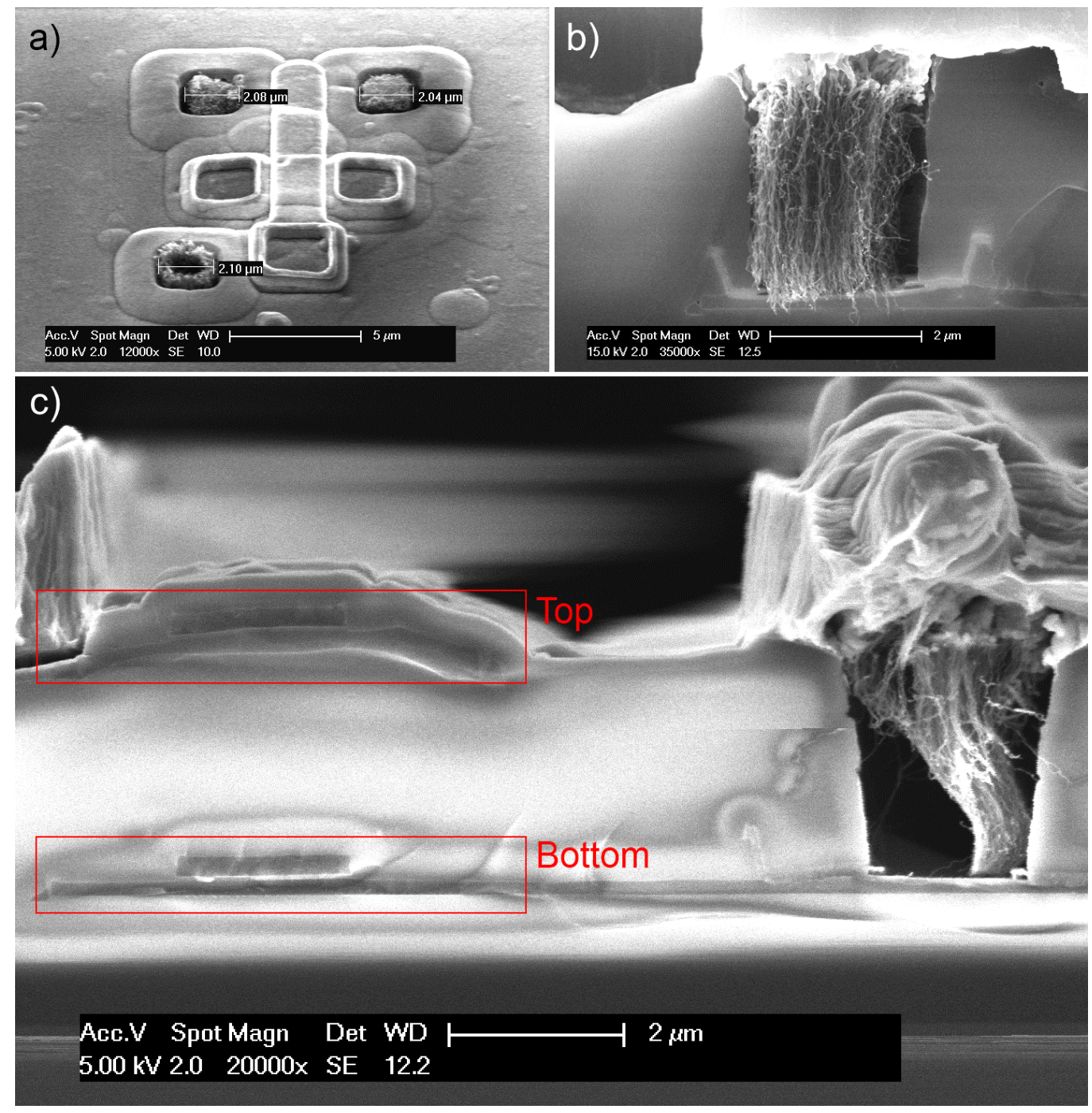

Figure 1: SEM images obtained from the wafer containing the 3D devices: a) top-view of 3D inverter after CNT growth displaying second layer transistor, b) detailed cross-section of a $2 \mu \mathrm{m}$ wide $\mathrm{CNT}$ via, the light area is the $\mathrm{Ti} / \mathrm{TiN}$ layer, c) cross-section of two-layer device showing the active areas (outlined by red squares) and CNT via. 


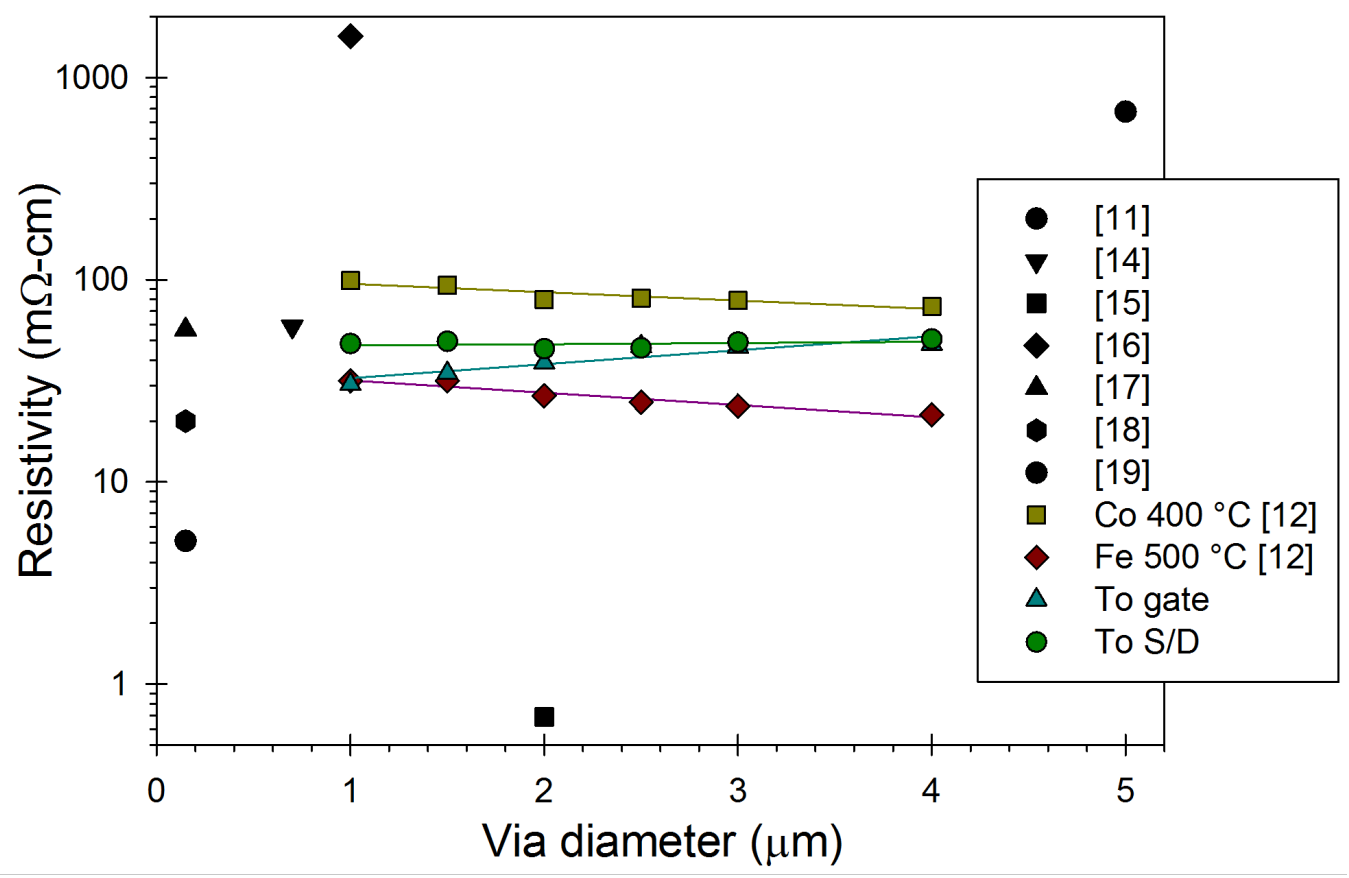

Figure 2: Calculated resistivity compared with values obtained before from CNT test vias without integrated electronics, and values from literature.

Table 1: Extracted average performance parameters for the 3D inverters.

\begin{tabular}{c|c|c|c|c|c}
$\begin{array}{c}\text { Bottom } \\
\text { transistor }\end{array}$ & $\begin{array}{c}\text { Via width } \\
{[\mu \mathrm{m}]}\end{array}$ & $\begin{array}{c}\mathrm{V}_{\mathrm{M}} \\
{[\mathrm{V}]}\end{array}$ & $\begin{array}{c}\text { Swing } \\
{[\mathrm{V}]}\end{array}$ & $\begin{array}{c}\mathrm{VNM}_{\mathrm{L}} \\
{[\mathrm{V}]}\end{array}$ & $\begin{array}{c}\mathrm{VNM}_{\mathrm{H}} \\
{[\mathrm{V}]}\end{array}$ \\
\hline \multirow{2}{*}{ PMOS } & 1 & $1.41 \pm 0.46$ & $4.58 \pm 0.61$ & $0.56 \pm 0.46$ & $3.30 \pm 0.56$ \\
& 2 & $1.91 \pm 0.33$ & $4.95 \pm 0.20$ & $1.18 \pm 0.45$ & $2.71 \pm 0.40$ \\
& 3 & $1.98 \pm 0.28$ & $4.91 \pm 0.32$ & $1.29 \pm 0.42$ & $2.61 \pm 0.35$ \\
\hline \multirow{2}{*}{ NMOS } & 1 & $2.07 \pm 0.88$ & $4.58 \pm 0.67$ & $1.63 \pm 0.98$ & $2.25 \pm 1.10$ \\
& 2 & $1.44 \pm 0.50$ & $4.88 \pm 0.33$ & $0.89 \pm 0.48$ & $3.19 \pm 0.63$ \\
& 3 & $1.23 \pm 0.45$ & $4.89 \pm 0.30$ & $0.66 \pm 0.40$ & $3.47 \pm 0.55$
\end{tabular}




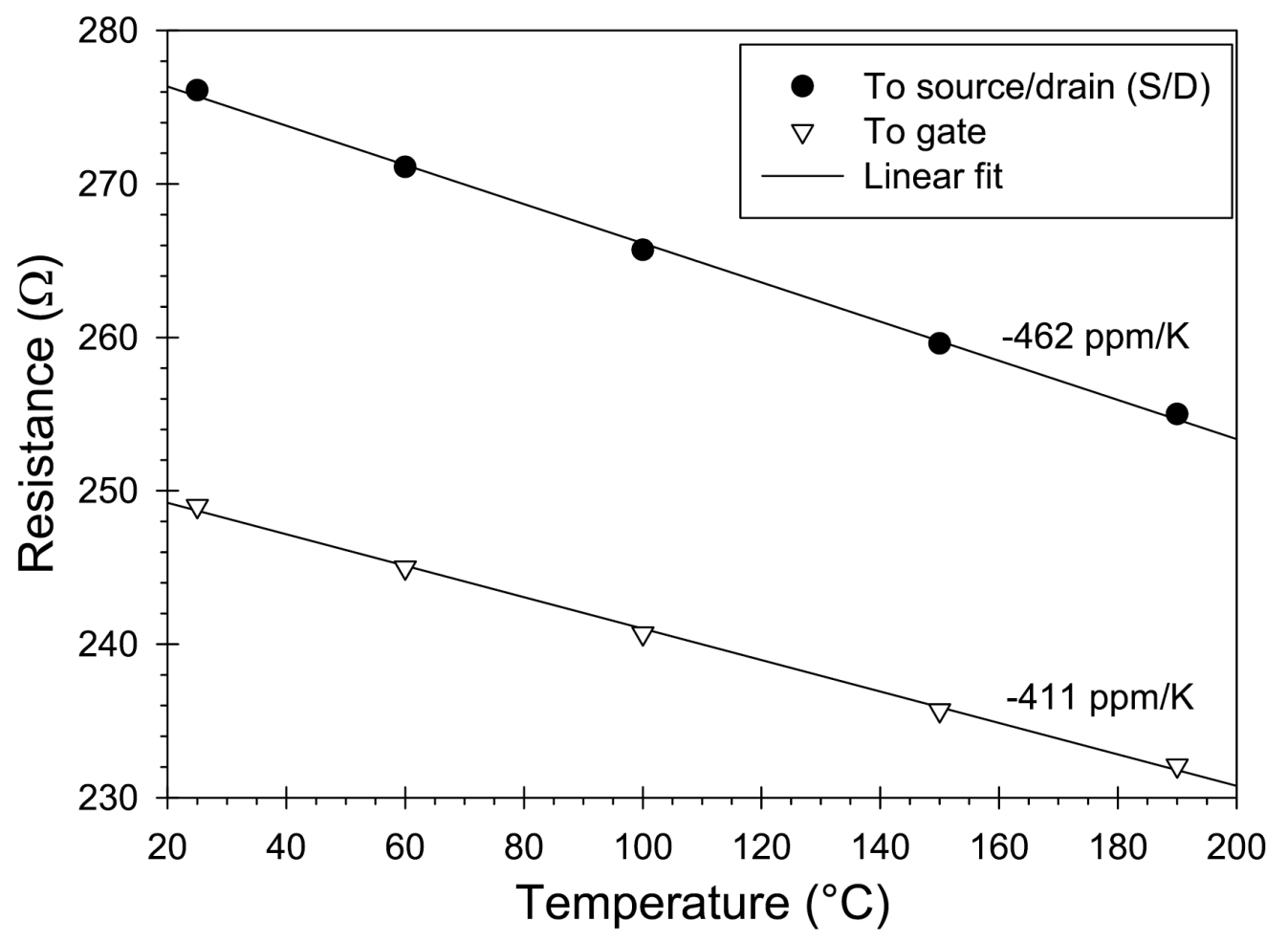

Figure 3: Resistance versus wafer temperature for typical $2 \mu \mathrm{m}$ wide vias. The solid lines indicate a linear least squares fit to the data points. 

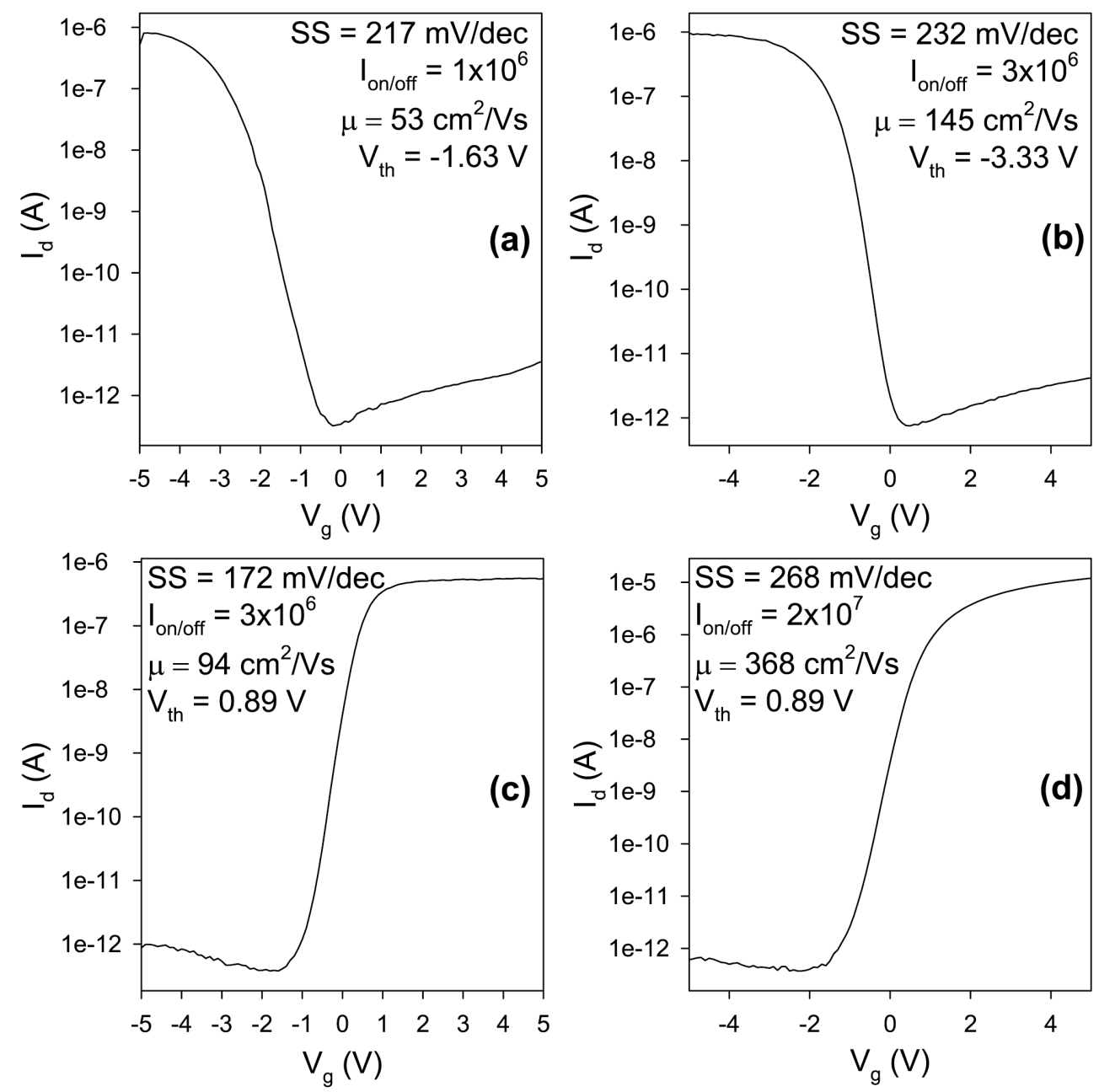

Figure 4: Typical $\mathrm{I}_{\mathrm{D}}-\mathrm{V}_{\mathrm{G}}$ characteristics for $\left.\operatorname{PMOS}(\mathrm{a}), \mathrm{b}\right)$ ) and NMOS (c), d)) transistors with $1 \mu \mathrm{m}(\mathrm{a}), \mathrm{c}))$ and $2 \mu \mathrm{m}(\mathrm{b}), \mathrm{d}))$ wide CNT vias. For the $2 \mu \mathrm{m}$ wide via, the on/off ratio, subtreshold slope (SS), mobility and threshold voltage are comparable to that of 3D IC without CNT reported previously [25]. $\mathrm{V}_{\mathrm{D}}=0.1 \mathrm{~V}$ for all measurements. 


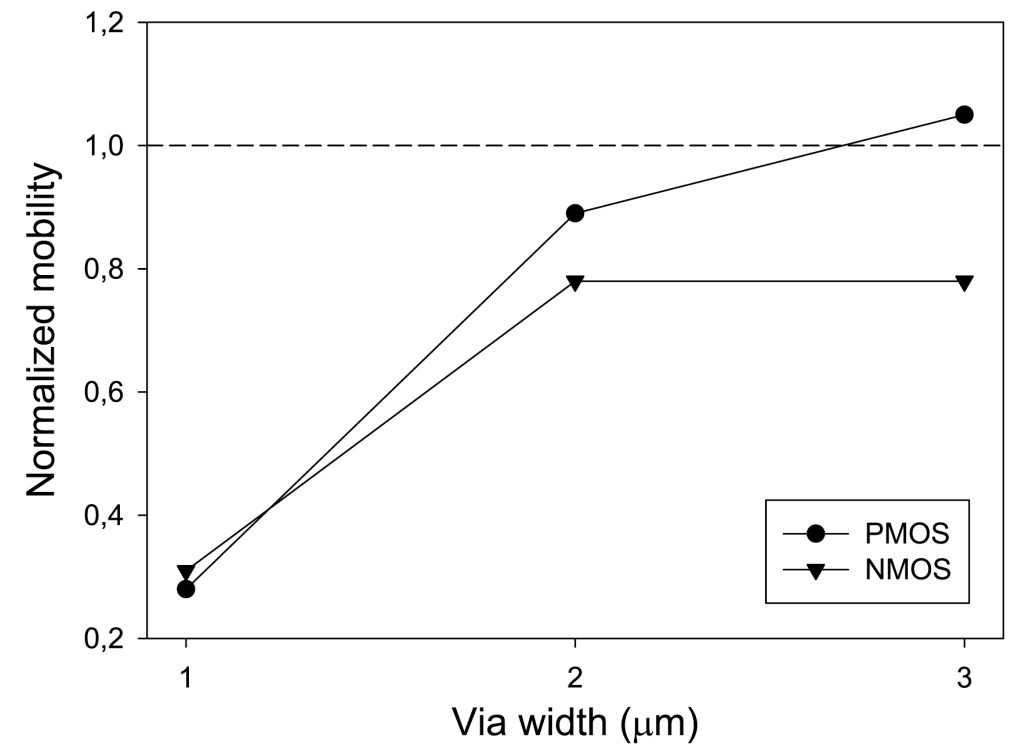

(a)

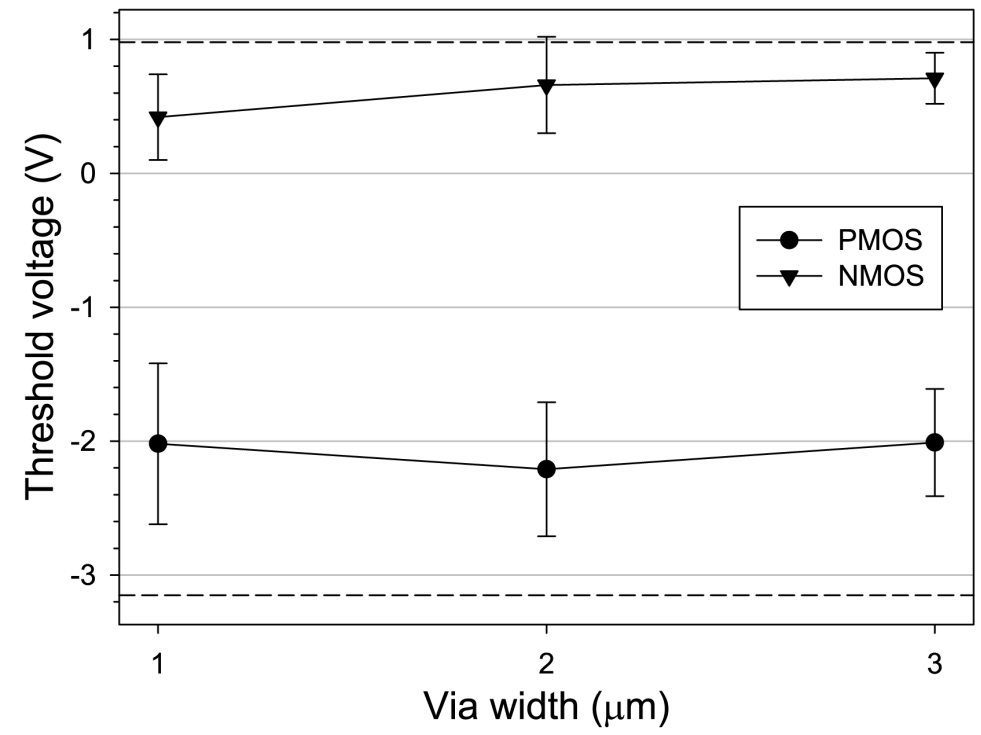

(b)

Figure 5: a) Average bottom transistor mobility (normalized to average top transistor mobility) against via width; b) bottom transistor threshold voltage versus width with the dashed lines indicating the top transistor $\mathrm{V}_{\text {th }}$. 


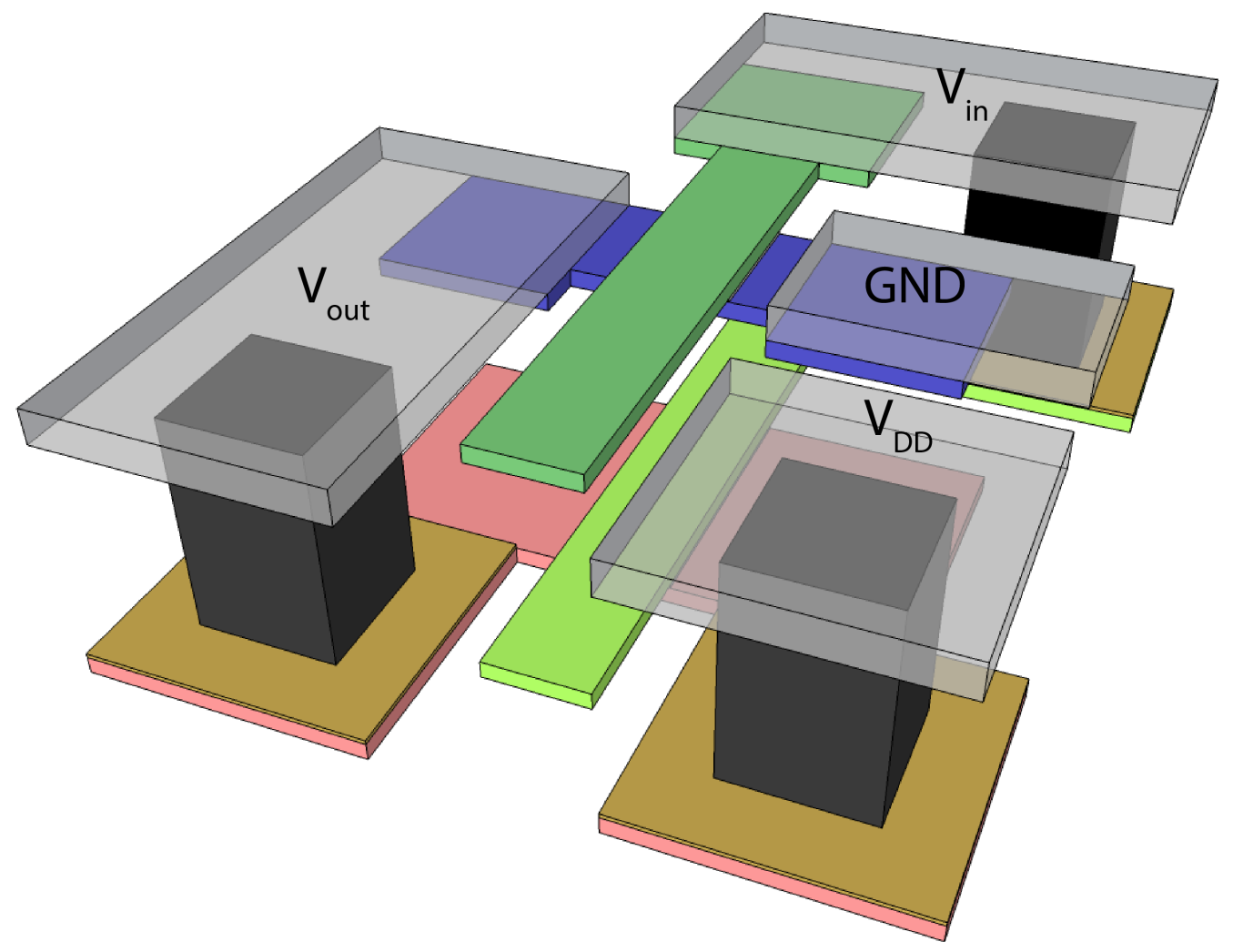

Figure 6: Design of a 3D SG-TFT inverter. The red and blue layer depict the p-type and $\mathrm{n}$-type $\mathrm{Si}$ islands, respectively, while the green areas are the gates. The brown areas are the $\mathrm{Ti} / \mathrm{TiN}$ pads for the catalyst support, the black areas the CNT bundles, and finally the grey areas the $\mathrm{Al}(1 \mathrm{Si})$ metallization. Oxide layers are omitted for clarity. 


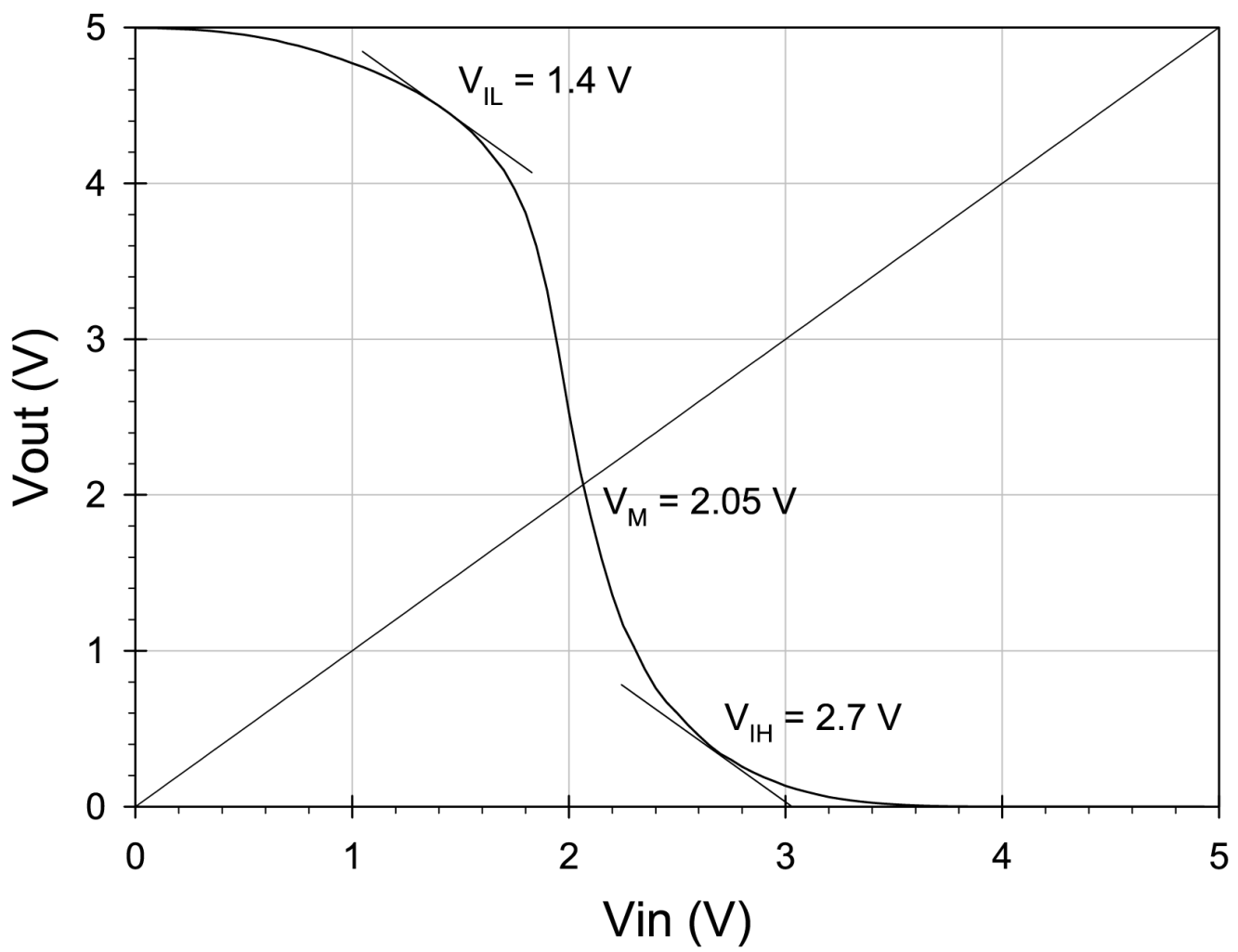

Figure 7: $\mathrm{V}_{\mathrm{in}}-\mathrm{V}_{\text {out }}$ characteristics of a PMOS bottom 3D inverter with $1 \mu \mathrm{m}$ wide vias. 


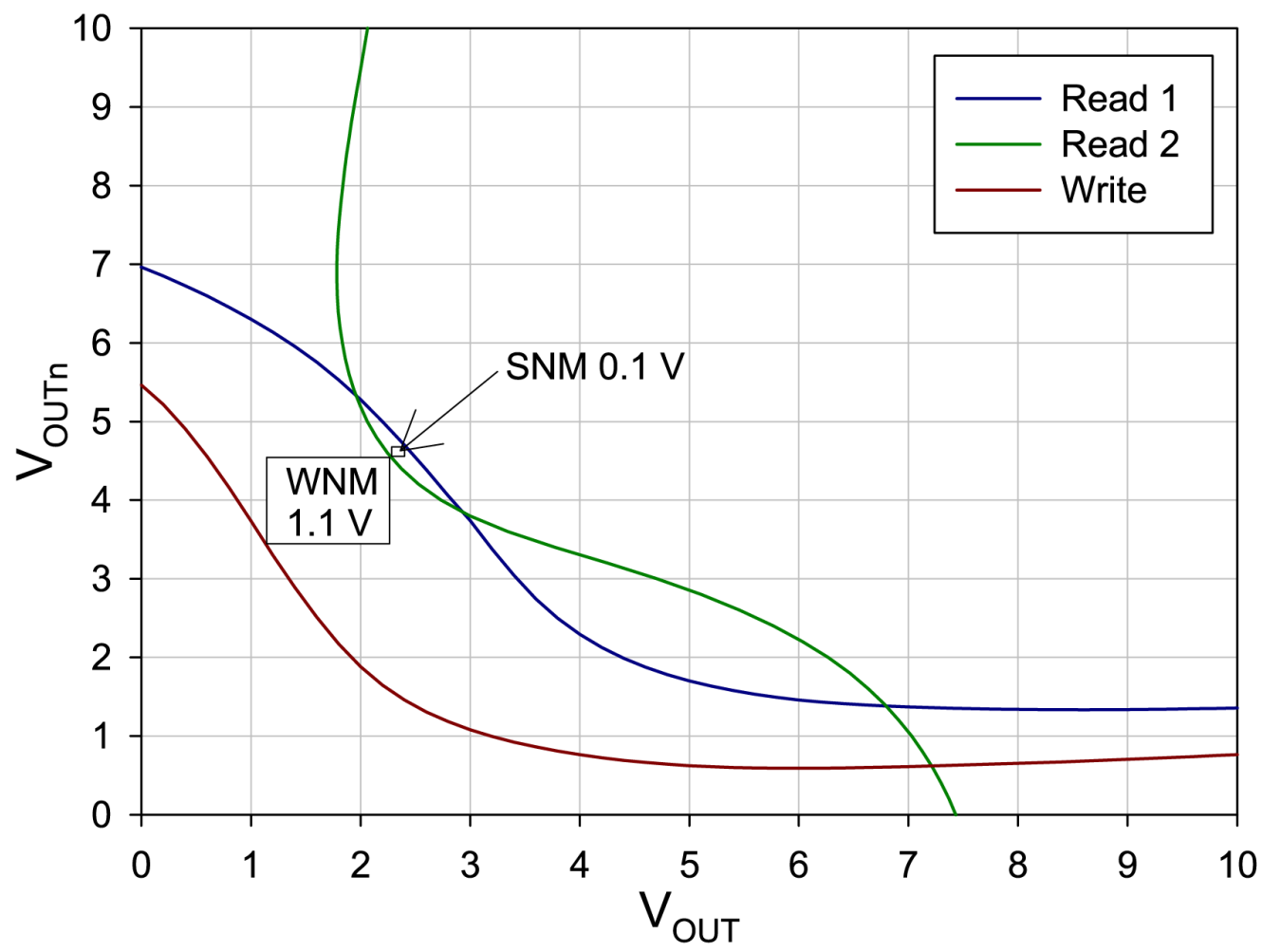

Figure 8: $\mathrm{V}_{\text {OUT }}-\mathrm{V}_{\text {OUTn }}$ characteristics of a 3D SG-TFT 6T-SRAM cell during read and write operations. 